\title{
FISHES OF GOBIIDAE FAMILY, RECORDED FROM THE RIVERS AND ESTUARIES OF BANGLADESH: SOME MORPHOMETRIC AND MERISTIC STUDIES
}

\author{
Gulshan Ara Latifa*, Abu Tweb Abu Ahmed, Md. Sagir Ahmed, Md. Mizanur \\ Rahman, Md. Asaduzzaman ${ }^{1}$, Md. Abu Obaida, Md. Muzammel Hossain ${ }^{1}$ \\ and Aparna Riti Biswas \\ Department of Zoology, University of Dhaka, Dhaka-1000, Bangladesh
}

\begin{abstract}
The study was carried out to determine morphometric and meristic characteristics of fish species belonging to Family: Gobiidae, and examined 42 specimens were collected from rivers and estuaries ecosystem in Bangladesh during January and February, 2015. Samples were found in fresh to brackish water and muddy habitats of estuary areas at Nijhum Island, Hatiya; junction of Meghna River and Bay of Bengal, and also recorded from Pasur River is near the Sundarbans in Bagerhat. Morphometric and meristic characteristics of Taenioides buchanani (Day 1873); Odontamblyopus rubicundus (Hamilton 1822); Trypauchen vagina (Bloch and Schneider 1801); Pseudapocryptes elongates (Cuvier 1816); Scartelaos histophorus (Valenciennes 1837); Apocryptes bato (Hamilton 1822) and Boleophthalmus boddarti (Pallas 1770) have been studied and comparatively described. This paper discusses the diagnostic characteristics of these fishes.
\end{abstract}

Key words: Gobiidae taxonomic study, fish, ecosystem.

\section{INTRODUCTION}

Gobies of the genus are also known as eel gobies which belong to the family Gobiidae, which alludes to their attenuated body shape. These species inhabits estuaries (Talwer and Jhingran 1991), shallow coastal water and rivers (Allen 1991). This group is morphologically unique among Gobiidae, having an elongate body, small eyes covered with skin and numerous distinct dermal folds, lobes, or barbels on the head. Fourteen nominal species were recognized in the genus Taenioidess. str. (Murdy and Randall 2002; hereafter referred to in this script as the genus "Taenioides"). In the north western Pacific, only the nominal species O. lacepedii sensu lato was reported to be widely distributed in the northern South China Sea, East China Sea, Yellow Sea, Gulf of Bohai and Araike Bay (Murdy and Shibukawa 2001, Shen 2008). Furthermore the difficulty of collecting eel gobies due to their subterranean habitat has led to their rarity in museum collections (Murdy and Randall 2002).

\footnotetext{
* Corresponding author: gulshan_al@yahoo.com. 1Department of Zoology, Jagannath University, Dhaka-1100, Bangladesh
}

(C) 2015 Zoological Society of Bangladesh DOI: 10.3329/bjz.v43i2.27389 
Gobiids are poorly explored in the freshwaters of Bangladesh. Only 18 species under 15 genera are recorded from Bangladesh (Rahman 2005). Due to their burying habits, specimens of Taenioides are not common in the collections and individually are normally not observed by fishery. The fishes of suborder Gobioidei belong to the largest group of those in present living Perciformes. There are about 2211 nominal species belonging to 270 genera of 9 families in the world (Nelson 2006) in the suborder. Gobies are distributed worldwide, in marine, estuarine and freshwater habitats. The American seven-spined goby includes 26 genera that represent $40 \%$ of New World gobiid genera and $50 \%$ of the species (Birdsong and Robins 1995, Ruber et al. 2003). Two species, Taenioides cirratus and T. snyderi, have been recorded from Japan (Jordan and Hubbs 1925, Tomiyama 1936, Akihito et al. 2002). Tomiyama (1936) recognized $T$. cirratusas one of the subspecies of $T$. gracilis, $T$. g. cirratus, which was distinguished from T. g. gracilis in having dermal folds on the head (Herre 1927). Such bimodality in characteristics is sometimes thought to reflect the existence of two or more reproductively isolated units in a single operational taxonomic unit (McDowall and Frankenberg 1981, Jones and van Parijs 1993). Although the identifications of four species need additional verifications, they were identified as T. anguillaris, T. snyderi, T. gracilis and T. cf. kentalleni, and the species name $T$. cirratusdoes not seem to be appropriate to any of four detected species (Kurita and Yoshino 2012).

The aim of this paper is need to more research on taxonomical records of the goby fish fauna from rivers and estuaries area in Bangladesh.

\section{MATERIAL AND METHODS}

Sampling sites and study period: Specimens were collected from Nijhum Island, Hatiya, south west area of the junction of Meghna River and Bay of Bengal, and adjacent areas of Pasur River is near the Sundarbans in Bagerhat during 20 January to 20 February, 2015 (Fig. 1 and 2). Nijhum Island incorporating an area of about $164 \mathrm{~km}^{2}$. This island raised on the estuary of the great Meghna river channel in the mouth of the Bay of Bengal. The geographical location is just 2 kilometers southwest of Hatia, Noakhali. Pasur River is near the Sundarbans in Bagerhat and a distributary of the Ganges. It continues the Rupsa River and it meets Shibsa River within the Sundarbans, Bangladesh.

Collection and Preservation: A total of 42 fish specimens were collected from fisherman within fishing time in water and muddy habitat. Fish materials identified were collected using commercial gill net, set beg net, bottom trawlers in water and digging tools. All counts and measurements were made from Gobiidae specimens preserved in $70 \%$ ethanol for taxonomic study. Some of these are mentioned following the comprehensive systematic accounts of the Inland Fishes of the Indian Region and Adjacent countries (Talwar and Jhingran 1991) and Freshwater Fishes of the World (Sterba 1962). 


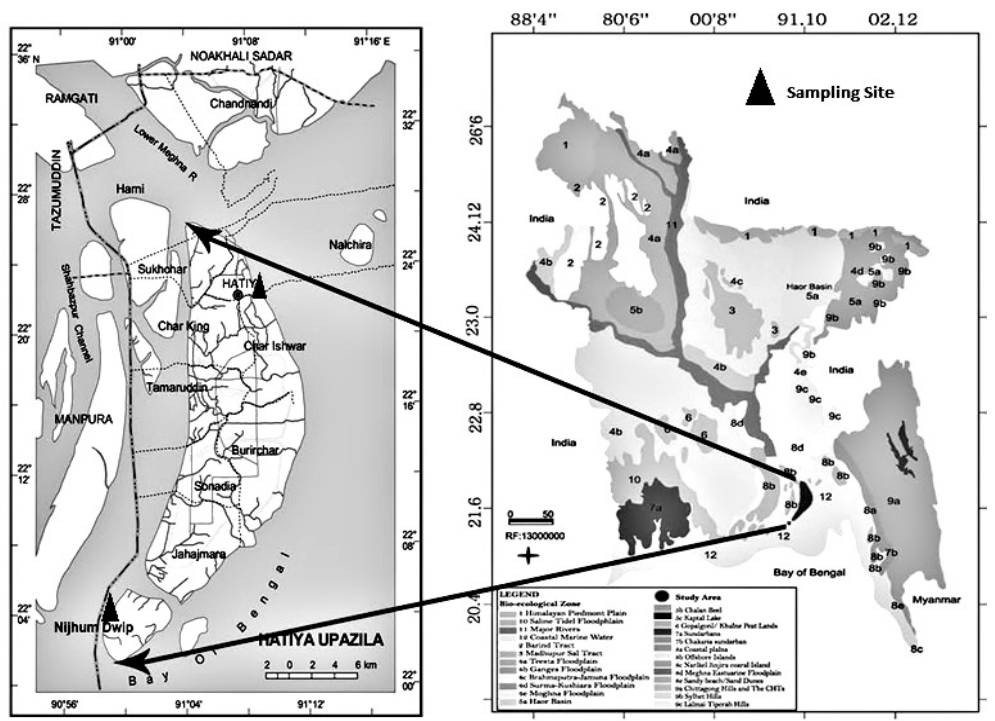

Fig. 1. Sampling sites at Hatia, Nijhum Island in the estuary area of Meghna River and adjacent area of mangrove forest of Bangladesh.

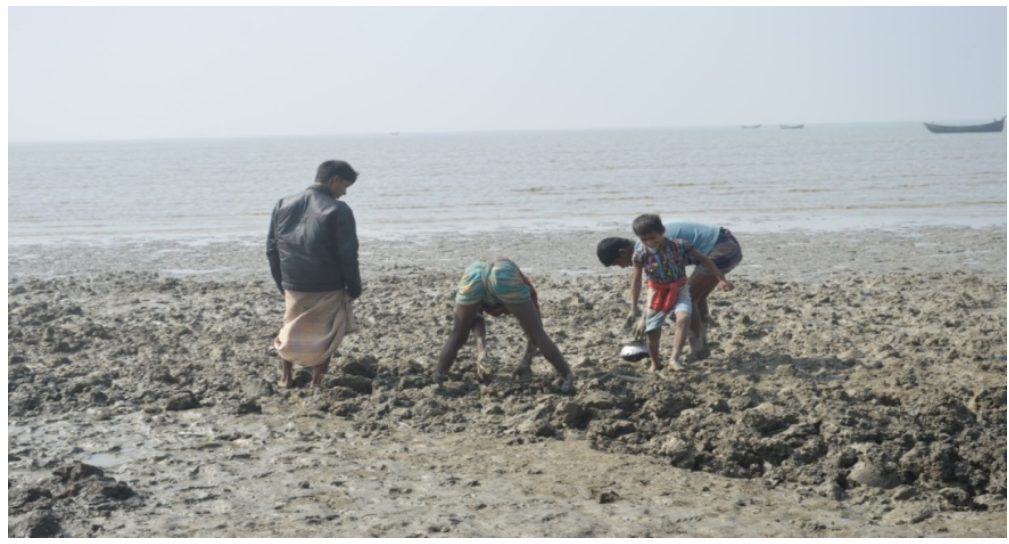

Fig. 2. Local fishermen are collecting Gobiidae specimens from muddy habitat at Nijhum Island.

The morphmetric and meristic measurements are followed by Murdy and Shibukawa (2001) and a total of 42 specimens were examined individually and recorded maximum size with average. The morphometric measurements are as follows of fish species i.e. $\mathrm{TL}=$ Total Length; $\mathrm{HL}=$ Head length; $\mathrm{P} 2 \mathrm{FB}=$ Pelvic Fin base; $\mathrm{PL}=$ Peduncle Length; $\mathrm{IOL}=$ Inter Orbital Length; $\mathrm{PD}=$ Peduncle Depth; $\mathrm{P} 1 \mathrm{FB}=$ Pectoral Fin Base PDL = Pre-Dorsal length; Po. OL = Post Orbital Length; $\mathrm{SALL}=$ Scales Above Lateral Line; $\mathrm{DFB}=$ Dorsal Fin Base; $\mathrm{SL}=$ Standard Length; Pr. $\mathrm{OL}=$ Pre Orbital Length; $\mathrm{SAL}=$ Scales Along Lateral Line; $\mathrm{SBL}=$ Scales Below Lateral Line; FL= Fork length; Pectoral Fin length = PECL; Pelvic Fin length = 
$\mathrm{PEL} ; \mathrm{ED}=$ Eye diameter; $\mathrm{AFB}=$ Anal fin Base; $\mathrm{BD}=$ Body Depth; $\mathrm{Br}=$ Branchiostegal Rays; D1, D2 $=1^{\text {st }}$ and $2^{\text {nd }}$ Dorsal Fin.

Meristic measurements are as follows: SL/TL; HL/SL; Pelvic Fin length (PEL)/SL; PEL/HL; Pectoral Fin length (PEC)/SL; PEC/HL; PEC/PEL; Body Depth (BD)/SL; Pre-Dorsal length (PDL)/SL; Inter Orbital Length (IOL)/SL.

\section{RESULTS AND DISCUSSION}

A total number of 7 specimens of Taenioides buchanani (Day 1873); 5 of Odontamblyopus rubicundus (Hamilton 1822); 6 of Trypauchen vagina (Bloch and Schneider 1801) species; 8 of Pseudapocryptes elongates (Cuvier 1816); 7 of Scartelaos histophorus (Valenciennes 1837); 4 of Apocryptes bato (Hamilton 1822) and 5 of Boleophthalmus boddarti (Pallas 1770) have counted individually and comparatively described below with distribution and original photographs (Table 1 and 2).

\section{Taenioides buchanani (Day 1873)}

Local Name: Chewa; English Name: Eel worm goby

Taxonomic position: Phylum: Chordata-chordates; Class: Actinopterygii- ray finned fishes, Spiny rayed fishes; Order: Perciformes - Perch like fishes; Family: Gobiidae - Gobies; Genus: Eel gobies; Species: Taenioides buchanani (Day 1873) (Plate.1.a).

Distribution: In the present study the species were found inhabits muddy bottoms of estuaries areas in Meghna river at Nijhum Dwip (Island) and GPS location $22^{\circ} 02^{\prime} 30.6^{\prime \prime} \mathrm{N}, 90^{\circ} 58^{\prime} 24.5^{\prime \prime} \mathrm{E}$. It was also found in Pasur River is near the Sundarbans in Bagerhat. Rahman (2005) recorded maximum size is $38.2 \mathrm{~cm}$ in total length from Meghna and Dakatia rivers near Chandpur. Takaki Kurita and Tetsuo Yoshino (2012) also recorded the species inhabits muddy bottoms of estuaries or shallow areas of seas in the Indo-Pacific Ocean, Japan.

Description : Morphometric measurements are as follows, Number of Fin Rays/Spines: Total dorsal fin rays 47; Pectoral Fin Rays 18; Pelvic Fin Rays 5; Anal Fin Rays 39; Anal fin narrowly jointed with caudal fin base, Caudal Fin Rays 14; Mouth position terminal; Branchiostegal Rays 4; Body measurement: Maximum Total Length $27.8 \mathrm{~cm}$ and standard length $23.7 \mathrm{~cm}$; Pre-Dorsal length $5.66 \mathrm{~cm}$; Body Depth $2.25 \mathrm{~cm}$; Head measurement: Eye diameter 1.1 $\mathrm{mm}$; Pre Orbital Length $10.2 \mathrm{~mm}$; Post Orbital Length 28.3mm; Inter Orbital Length $7.6 \mathrm{~mm}$; Fin base length: $1^{\text {st }}$ Dorsal Fin Base length $185 \mathrm{~mm}$; Pectoral Fin Base Length $6.7 \mathrm{~mm}$ and Pectoral Fin length $10.5 \mathrm{~mm}$; Pelvic Fin base length $10.4 \mathrm{~mm}$ and Pelvic Fin length $26.1 \mathrm{~mm}$; Anal fin Base length $13.5 \mathrm{~mm}$ (Plate.1.a).

Body is reddish inferiorly, pectorals and pelvics yellowish color. Head and body compressed a band of villiform teeth on each jaw behind the outer row of canines. A few rudimentary scales on the posterior part of the body, Eye rudimentary, but distinct, covered by skin. 
Meristic measurements are as follows: $\mathrm{SL} / \mathrm{TL}=0.852 ; \mathrm{HL} / \mathrm{SL}=0.199 ;$ Pelvic Fin length $(\mathrm{PEL}) / \mathrm{SL}=0.110 ; \mathrm{PEL} / \mathrm{HL}=0.552 ;$ Pectoral Fin length $(\mathrm{PEC}) / \mathrm{SL}=$ 0.443; PEC $/ \mathrm{HL}=0.222 ; \mathrm{PEC} / \mathrm{PEL}=0.402$; Body Depth $(\mathrm{BD}) / \mathrm{SL}=0.094$; PreDorsal length $(\mathrm{PDL}) / \mathrm{SL}=0.238$; Inter Orbital Length $(\mathrm{IOL}) / \mathrm{SL}=0.032$ (Plate.1.a).

\section{Trypauchen vagina (Bloch and Schneider 1801)}

Local name: Sada Chewa; English name: Burrowing Goby

Taxonomic position: Phylum: Chordata-chordates; Class: Actinopterygii-ray finned fishes, spiny rayed fishes; Order: Perciformes- perch like fishes; Family: Gobiidae - true gobies, gobies, Genus: Trypauchen Cuvier and Valenciennes, 1837; Species: Trypauchen vagina (Bloch and Schneider 1801) (Plate.1.b).

Distribution: Trypauchen vagina (Bloch and Schneider 1801) species were found inhabits holes beneath tide pools and mud habitats in the near shore areas of Hatiya and Nijhum Island area of junction between Meghna River and Sea and GPS location $22^{\circ} 02^{\prime} 30.6^{\prime \prime}, 90^{\circ} 58^{\prime} 24.5^{\prime \prime} \mathrm{E}$. Also it was recorded in Pasur River is near the Sundarbans in Bagerhat, Mongla $22.4833^{\circ} \mathrm{N}, 89.6083^{\circ} \mathrm{E}$; Bhola and Cox's Bazaar estuaries area.

Description: Morphometric measurements are as follows, Number of Fin Rays/Spines: Total dorsal fin rays 50-57; Pectoral Fin Rays 17; Pelvic Fin Rays 12; Anal Fin Rays 44-50; Caudal Fin Rays 14; Mouth position Superior; Branchiostegal Rays 5; Body measurement: Maximum Total Length $14.4 \mathrm{~cm}$ and standard length $12.3 \mathrm{~cm}$; Pre-Dorsal length $2.51 \mathrm{~cm}$; Body Depth $1.34 \mathrm{~cm}$; Head measurement: Eye diameter $2.0 \mathrm{~mm}$; Pre Orbital Length $5.4 \mathrm{~mm}$; Post Orbital Length $14.0 \mathrm{~mm}$; Inter Orbital Length $3.6 \mathrm{~mm}$; Fin base length: 1st Dorsal Fin Base length $96 \mathrm{~mm}$; Pectoral Fin Base Length $4.2 \mathrm{~mm}$ and Pectoral Fin length $7.2 \mathrm{~mm}$; Pelvic Fin base length $2.9 \mathrm{~mm}$ and Pelvic Fin length $8.2 \mathrm{~mm}$; Anal fin Base length $58 \mathrm{~mm}$; Scales Above Lateral Line 75-85; Scales Below Lateral Line 612/2 Scales along lateral line 61/2 (Plate.1.b).

Body is pinky-white, compressed elongate and eyes blackish; barbell absent on head; Cycloid scales; Teeth in both jaws and caninoid; gill opening moderate; Pectoral short conical. Dorsal and anal fins grey; dorsal and anal connected with caudal fin; the species is not listed Endangered in the Red List of IUCN Bangladesh (2000).

Meristic measurements are as follows: $\mathrm{SL} / \mathrm{TL}=0.854$; HL/SL $=0.203$; Pelvic Fin length $(\mathrm{PEL}) / \mathrm{SL}=0.066 ; \mathrm{PEL} / \mathrm{HL}=0.328 ;$ Pectoral Fin length $(\mathrm{PEC}) / \mathrm{SL}=$ $0.058 ; \mathrm{PEC} / \mathrm{HL}=0.288 ; \mathrm{PEC} / \mathrm{PEL}=0.878$; Body Depth $(\mathrm{BD}) / \mathrm{SL}=0.108$; PreDorsal length $(\mathrm{PDL}) / \mathrm{SL}=0.204$; Inter Orbital Length $(\mathrm{IOL}) / \mathrm{SL}=0.029$ (Plate. $1 . \mathrm{b})$. 

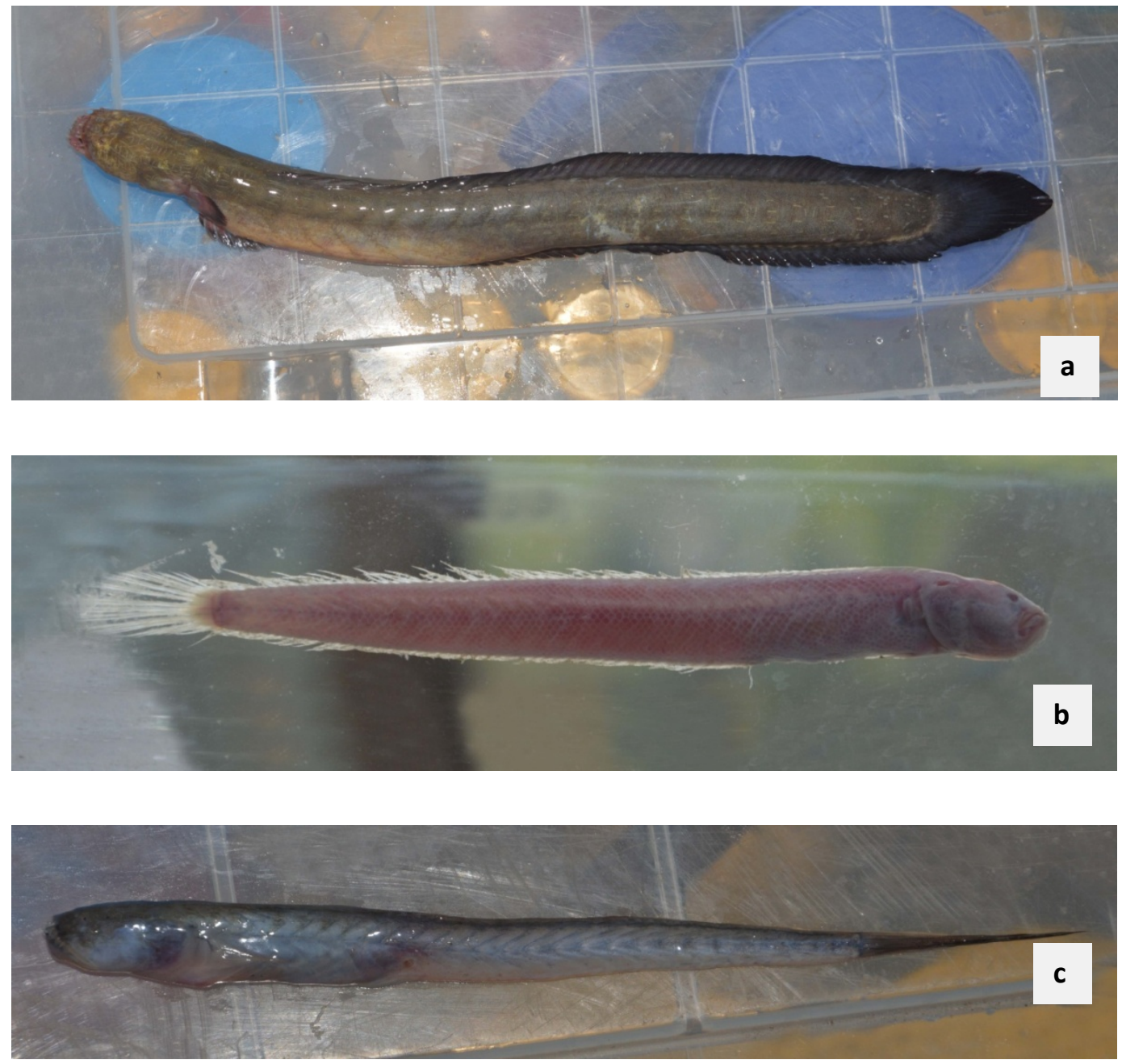

Plate 1: a) Taenioides buchanani (Day 1873); b) Trypauchen vagina (Bloch and Schneider 1801); c) Odontamblyopus rubicundus (Hamilton 1822). 

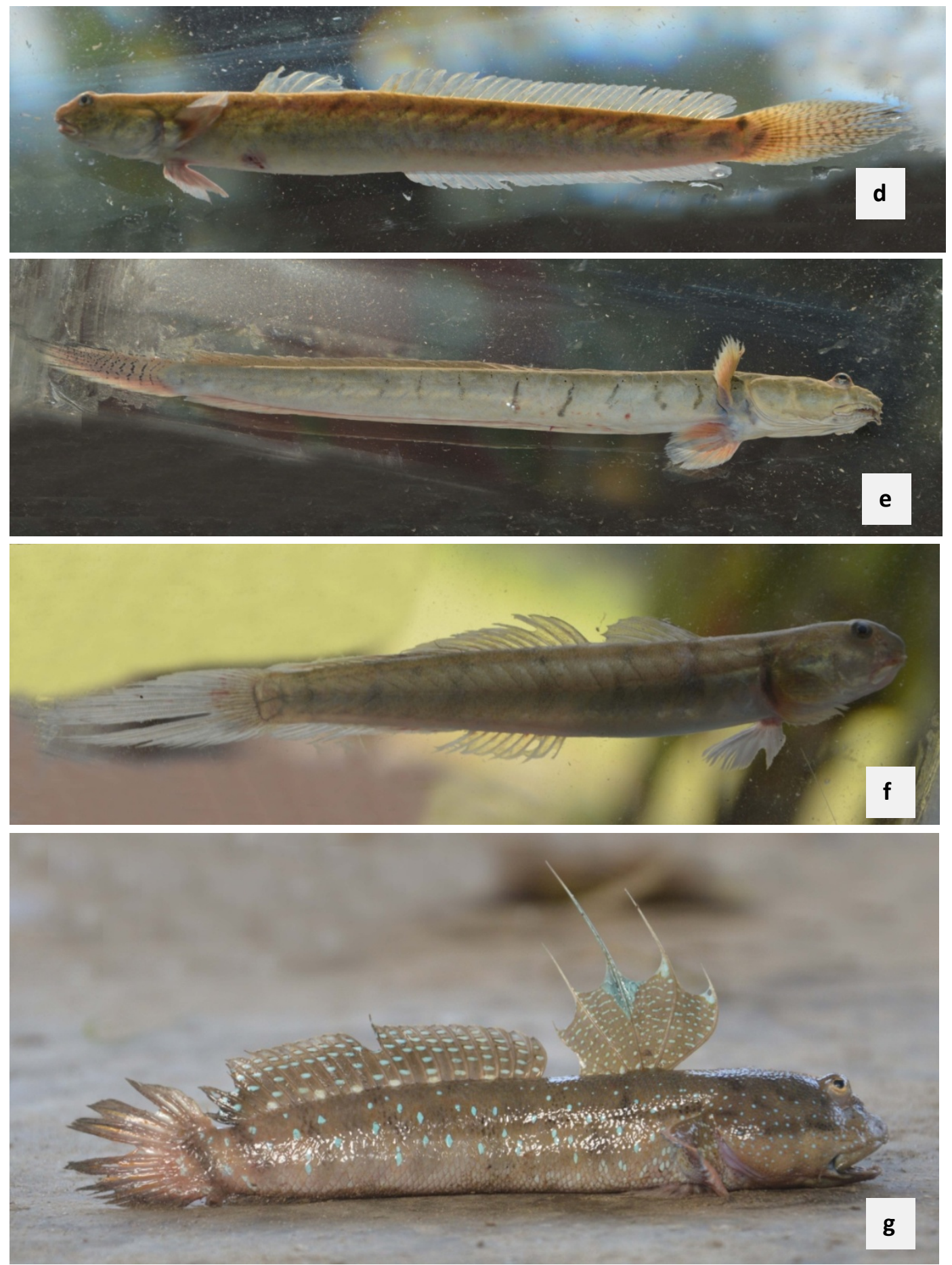

Plate 2: d) Pseudapocryptes elongates (Cuvier 1816); e) Scartelaos histophorus (Valenciennes 1837); f) Apocryptes bato (Hamilton, 1822); g) Boleophthalmus boddarti (Pallas 1770); 
Table 1. Average body, head and fin base measurement of Gobi fishes

\begin{tabular}{|c|c|c|c|c|c|c|c|c|c|c|c|c|c|c|c|c|c|c|c|c|c|}
\hline \multirow{3}{*}{$\begin{array}{l}\text { SI } \\
\text { No }\end{array}$} & \multirow{3}{*}{ Scientific name } & \multicolumn{7}{|c|}{ Body measurement (Average) } & \multicolumn{5}{|c|}{ Head measurement(Average) } & \multicolumn{5}{|c|}{ Fin base length(mm)(Average) } & \multicolumn{3}{|c|}{ No of scales } \\
\hline & & \multirow{2}{*}{$\begin{array}{c}\mathrm{TL} \\
(\mathrm{cm})\end{array}$} & \multirow{2}{*}{$\begin{array}{l}\mathrm{FL} \\
(\mathrm{cm})\end{array}$} & \multirow{2}{*}{$\begin{array}{c}\mathrm{SL} \\
(\mathrm{cm})\end{array}$} & \multirow{2}{*}{$\begin{array}{l}\text { PDL } \\
\text { (cm) }\end{array}$} & \multirow{2}{*}{$\begin{array}{l}B D \\
(\mathrm{~cm})\end{array}$} & \multirow{2}{*}{$\begin{array}{l}\mathrm{PL} \\
(\mathrm{cm})\end{array}$} & \multirow{2}{*}{$\begin{array}{l}\text { PD } \\
(\mathrm{cm})\end{array}$} & \multirow{2}{*}{$\begin{array}{c}\mathrm{HL} \\
(\mathrm{cm})\end{array}$} & \multirow{2}{*}{$\begin{array}{c}\text { ED } \\
(\mathrm{mm})\end{array}$} & \multirow{2}{*}{$\begin{array}{l}\text { Pr.OL } \\
(\mathrm{mm})\end{array}$} & \multirow{2}{*}{$\begin{array}{l}\begin{array}{l}\text { Po.0l } \\
(\mathrm{mm})\end{array}\end{array}$} & \multirow{2}{*}{$\begin{array}{l}\text { IOL } \\
(\mathrm{mm})\end{array}$} & \multicolumn{2}{|c|}{ DFB } & \multirow[t]{2}{*}{ P1FB } & \multirow[t]{2}{*}{ Р2FB } & \multirow[t]{2}{*}{ AFB } & \multirow[t]{2}{*}{ SALL } & \multirow[t]{2}{*}{ SAbl } & \multirow[t]{2}{*}{ SBL } \\
\hline & & & & & & & & & & & & & & $1^{2:}$ & $2^{-6}$ & & & & & & \\
\hline 1 & $\begin{array}{l}\text { Taenioides } \\
\text { buchanani(Day, } \\
\text { 1873) }\end{array}$ & 27.8 & & 23.7 & 5.66 & 2.25 & & & 4.72 & 1.1 & 10.2 & 28.3 & 7.6 & 185 & & $\begin{array}{c}6.7 \\
L-10.5\end{array}$ & $\begin{array}{c}10.4 \\
\text { L-26.1 }\end{array}$ & 13.5 & & & \\
\hline 2 & $\begin{array}{l}\text { Trypauchen vagina } \\
\text { (Bloch and } \\
\text { Schneider, 1801) }\end{array}$ & 14.4 & & 12.3 & 2.51 & 1.34 & & & 2.5 & 2.0 & 5.4 & 14.0 & 3.6 & 96.0 & & $\begin{array}{c}4.2 \\
\text { L- } 7.2\end{array}$ & $\begin{array}{c}2.9 \\
L-8.2\end{array}$ & 78 & $75-85$ & 612 & $6 / 2$ \\
\hline 3 & $\begin{array}{l}\text { Odontamblyopus } \\
\text { rubicundus } \\
\text { (Hamilton, 1822) }\end{array}$ & 24.2 & & 19.5 & 4.12 & 1.47 & & & 3.75 & 1.0 & 8.2 & 21.5 & 6.8 & 16.8 & & $\begin{array}{c}6.5 \\
\mathrm{~L}- \\
18.4\end{array}$ & $\begin{array}{c}6.3 \\
L-21.6\end{array}$ & 127 & & & \\
\hline 4 & $\begin{array}{l}\text { Pseudapocryptes } \\
\text { elongates (Cuvier, } \\
1816 \text { ) }\end{array}$ & 15 & & 12 & 3.51 & 1.68 & 0.51 & 0.9 & 2.61 & 3 & 4.5 & 14.9 & 3.7 & $\begin{array}{l}13.2 \\
\text { L- } \\
13.1\end{array}$ & 61.5 & $\begin{array}{l}6.9 \\
L- \\
13.8\end{array}$ & $\begin{array}{c}4.6 \\
\text { L- } 12.2\end{array}$ & 51.6 & & & \\
\hline 5 & $\begin{array}{l}\text { Scartelaos } \\
\text { histophorus } \\
\text { (Valenciennes } \\
\text { 1837) }\end{array}$ & 14.8 & & 11.4 & 3.67 & 1.17 & 0.22 & 0.63 & 2.84 & 4.5 & 6.2 & 15.1 & 2.6 & $\begin{array}{l}5.7 \\
\text { L- } 44\end{array}$ & 62.3 & $\begin{array}{c}7.3 \\
L- \\
13.3\end{array}$ & $\begin{array}{c}4.7 \\
\text { L- }-12.9\end{array}$ & 56.5 & & & \\
\hline 6 & $\begin{array}{l}\text { Apocryptes bato } \\
\text { (Hamilton, 1822) }\end{array}$ & 13 & & 8.6 & 2.5 & 1.12 & 0.52 & 0.7 & 1.91 & 3.6 & 4.5 & 9.2 & 1.8 & 13.7 & 41.3 & $\begin{array}{c}5.9 \\
L \cdot \\
10.8\end{array}$ & $\begin{array}{c}3.8 \\
\text { L- } 10.8\end{array}$ & 35.7 & $60-80$ & & \\
\hline 7 & $\begin{array}{l}\text { Boleophthalmus } \\
\text { boddarti(Pallas, } \\
1770)\end{array}$ & 7.17 & & 5.78 & 2.3 & 7.6 & 0.3 & 0.41 & 1.89 & 3.5 & 4.2 & 9.5 & 1.7 & $\begin{array}{c}3.1 \\
L- \\
141\end{array}$ & 24.5 & $\begin{array}{c}5.3 \\
L- \\
11.0\end{array}$ & $\begin{array}{c}3.7 \\
L-9.3\end{array}$ & 22.9 & & & \\
\hline
\end{tabular}


Table 2. Average number of fin/spines measurements with mouth position of Gobi fishes

\begin{tabular}{|c|c|c|c|c|c|c|c|c|c|c|}
\hline \multirow{3}{*}{$\begin{array}{l}\text { SI } \\
\text { No }\end{array}$} & \multirow[t]{3}{*}{ Scientific name } & \multirow[t]{3}{*}{$\mathrm{Br}$} & \multicolumn{6}{|c|}{ Number of Fin Rays/Spines (Average) } & \multirow{3}{*}{$\begin{array}{l}\text { Mouth } \\
\text { Position }\end{array}$} & \multirow[t]{3}{*}{ Body Color } \\
\hline & & & \multicolumn{2}{|c|}{ DFR/DFS } & \multirow[t]{2}{*}{ P1FR } & \multirow[t]{2}{*}{ P2FR } & \multirow[t]{2}{*}{ AFR } & \multirow[t]{2}{*}{ CFR } & & \\
\hline & & & D1 & $\mathrm{D} 2$ & & & & & & \\
\hline 1 & Taenioides buchanani (Day, 1873) & 4 & 47 & & 18 & 5 & 39 & 14 & Terminal & \\
\hline 2 & $\begin{array}{l}\text { Trypauchen vagina (Bloch and Schneider, } \\
\text { 1801) }\end{array}$ & 5 & $\begin{array}{l}50- \\
57\end{array}$ & & 17 & 12 & $44-50$ & 14 & Superior & Radish \\
\hline 3 & $\begin{array}{l}\text { Odontamblyopus rubicundus (Hamilton, } \\
\text { 1822) }\end{array}$ & 5 & 42 & & 31 & 6 & 36 & $9+9$ & Terminal & Brownish \\
\hline 4 & Pseudapocryptes elongates (Cuvier, 1816) & 2 & 5 & 32 & 17 & 5 & 29 & $11+11$ & Sub-Terminal & \\
\hline 5 & $\begin{array}{l}\text { Scartelaos histophorus (Valenciennes } \\
1837 \text { ) }\end{array}$ & 3 & 7 & 28 & 17 & 12 & 26 & $9+9$ & Sub-Terminal & \\
\hline 6 & Apocryptes bato (Hamilton, 1822) & $4 / 3$ & 5 & 21 & 23 & 12 & 22 & $10+10$ & Sub-Terminal & Transparent \\
\hline 7 & Boleophthalmus boddarti (Pallas, 1770) & 3 & 5 & 20 & 18 & 12 & 21 & $9+9$ & Sub-Terminal & \\
\hline
\end{tabular}




\section{Odontamblyopus rubicundus (Hamilton 1822)}

Local name: Lal Chewa; English Name: Rubicundus Eel goby

Taxonomic position: Phylum: Chordata-chordates; Class: Actinopterygii-ray finned fishes, spiny rayed fishes; Order: Perciformes- perch like fishes; Family: Gobiidae - true gobies, gobies, Genus: Odontamblyopus (Bleeker 1874); Species: Odontamblyopus rubicundus (Hamilton 1822) (Plate.1.c).

Distribution: Odontamblyopus rubicundus (Hamilton, 1822) species were found inhabits holes beneath tide pools and mud habitats in the near shore areas of Nijhum Island area of junction Meghna River and Bay of Bengal. and GPS location $22^{\circ} 02^{\prime} 30.6^{\prime \prime} \mathrm{N}, 90^{\circ} 58^{\prime} 24.5^{\prime \prime} \mathrm{E}$. Also it was recorded in southwestern at Bhola Island. Rahman, 2005 recorded largest size is $29 \mathrm{~cm}$ in total length from Dakatia River near Chandpur. Murdy and Shibukawa (2001) also recorded the species in mud habitats along the coast of the Indo-West Pacific.

Description : Morphometric measurements are as follows, Number of Fin Rays/Spines: Total dorsal fin rays 42; Pectoral Fin Rays 31; Pelvic Fin Rays 6; Anal Fin Rays 36; Anal fin narrowly jointed with caudal fin base, Caudal Fin Rays 9+9; Mouth position terminal; Branchiostegal Rays 5; Body measurement: Maximum Total Length $24.2 \mathrm{~cm}$ and standard length $19.5 \mathrm{~cm}$; Pre-Dorsal length $4.12 \mathrm{~cm}$; Body Depth $1.47 \mathrm{~cm}$; Head measurement: Eye diameter 1.0 $\mathrm{mm}$; Pre Orbital Length $8.2 \mathrm{~mm}$; Post Orbital Length $21.5 \mathrm{~mm}$; Inter Orbital Length $6.8 \mathrm{~mm}$; Fin base length: 1st Dorsal Fin Base length $16.8 \mathrm{~mm}$; Pectoral Fin Base Length $6.5 \mathrm{~mm}$ and Pectoral Fin length $18.4 \mathrm{~mm}$; Pelvic Fin base length $6.3 \mathrm{~mm}$ and Pelvic Fin length $21.6 \mathrm{~mm}$; Anal fin Base length $127 \mathrm{~mm}$ (Plate.1.c).

Head sub-cylindrical, body is elongated, compressed, eel-like; three short barbels on each side below the head, eyes small, Teeth in both jaws and caninoid, Scales rudimentary, Pectoral, pelvic, dorsal and anal fins reddish, Caudal blackish; Dorsal and anal fins separated from the caudal, Caudal fin pointed.

Meristic measurements are as follows: $\mathrm{SL} / \mathrm{TL}=0.805 ; \mathrm{HL} / \mathrm{SL}=0.192$; Pelvic Fin length $(\mathrm{PEL}) / \mathrm{SL}=0.110 ; \mathrm{PEL} / \mathrm{HL}=0.576$; Pectoral Fin length $(\mathrm{PEC}) / \mathrm{SL}=$ 0.0943; PEC $/ \mathrm{HL}=0.490 ; \mathrm{PEC} / \mathrm{PEL}=0.851$; Body Depth $(\mathrm{BD}) / \mathrm{SL}=0.075$; PreDorsal length $(\mathrm{PDL}) / \mathrm{SL}=0.211$; Inter Orbital Length $(\mathrm{IOL}) / \mathrm{SL}=0.034$ (Plate.1.c).

\section{Pseudapocryptes elongates (Cuvier, 1816)}

Local name: Chewa; English Name: Pointed-tailed Goby

Taxonomic position: Phylum: Chordata-chordates; Class: Actinopterygii-ray finned fishes, spiny rayed fishes; Order: Perciformes- perch like fishes; Family: Gobiidae - true gobies, gobies, Genus: Pseudapocryptes Bleeker 1874; Species: Pseudapocryptes elongates (Cuvier 1816) (Plate.2.d). 
Distribution: In the present study the species were found inhabits muddy bottoms at Nijhum Island and estuaries areas of junction between Meghna River and Bay of Bangel, Hatiya. GPS location $22^{\circ} 02^{\prime} 30.6^{\prime \prime} \mathrm{N}, 90^{\circ} 58^{\prime} 24.5^{\prime \prime} \mathrm{E}$. Rahman 2005 recorded maximum size is $21 \mathrm{~cm}$ in total length from Meghan River near Chandpur area.

Description: Morphometric measurements are as follows, Number of Fin Rays/Spines: $1^{\text {st }}$ dorsal fin ray 5, $2^{\text {nd }}$ dorsal fin ray 32; Pectoral Fin Rays 17; Pelvic Fin Rays 5; Anal Fin Rays 29; Caudal Fin Rays 22; Mouth position horizontal; Branchiostegal Rays 2; Body measurement: Maximum Total Length $15 \mathrm{~cm}$ and standard length $12 \mathrm{~cm}$; Pre-Dorsal length $3.51 \mathrm{~cm}$; Body Depth 1.68 $\mathrm{cm}$; Peduncle Length $0.51 \mathrm{~cm}$; Peduncle Depth $0.9 \mathrm{~cm}$. Head measurement: Eye diameter $3 \mathrm{~mm}$; Pre Orbital Length $4.5 \mathrm{~mm}$; Post Orbital Length $14.9 \mathrm{~mm}$; Inter Orbital Length $3.7 \mathrm{~mm}$; Fin base length: $1^{\text {st }}$ Dorsal Fin Base length $13.2 \mathrm{~mm}$; $1^{\text {st }}$ Dorsal Fin length $13.1 \mathrm{~mm}, 2^{\text {nd }}$ Dorsal Fin Base length $61.5 \mathrm{~mm}$; Pectoral Fin Base Length $6.9 \mathrm{~mm}$ and Pectoral Fin length $13.8 \mathrm{~mm}$; Pelvic Fin base length $4.6 \mathrm{~mm}$ and Pelvic Fin length $12.2 \mathrm{~mm}$; Anal fin Base length $51.6 \mathrm{~mm}$ (Plate.2.d).

Mouth type horizontal, body elongate, cylindrical anteriorly and compressed posteriorly Scales cycloid, Teeth in one row on each jaw, teeth horizontal in the lower jaw with canine on each side in mouth, Maxillary extends, Tongue rounded, Caudal long, pointed, Second dorsal and caudal fins with several rows of fine spots. A black mark at the upper base of the caudal (Rahman 2005).

Meristic measurements are as follows: $\mathrm{SL} / \mathrm{TL}=0.8 ; \mathrm{HL} / \mathrm{SL}=0.217$; Pelvic Fin length $(\mathrm{PEL}) / \mathrm{SL}=0.101 ; \mathrm{PEL} / \mathrm{HL}=0.467 ;$ Pectoral Fin length $(\mathrm{PEC}) / \mathrm{SL}=$ $0.115 ; \mathrm{PEC} / \mathrm{HL}=0.528 ; \mathrm{PEC} / \mathrm{PEL}=1.131$; Body Depth $(\mathrm{BD}) / \mathrm{SL}=0.14$; PreDorsal length $(\mathrm{PDL}) / \mathrm{SL}=0.2925$; Inter Orbital Length $(\mathrm{IOL}) / \mathrm{SL}=$ 0.030(Plate.2.d).

\section{Scartelaos histophorus (Valenciennes 1837)}

Local Name: Dahuk ; English Name: Walking Goby

Taxonomic position: Phylum: Chordata-chordates; Class: Actinopterygii-ray finned fishes, spiny rayed fishes; Order: Perciformes- perch like fishes; Family: Gobiidae - true gobies, gobies, Genus: Scartelaos (Swainson 1839); Species: Scartelaos histophorus (Valenciennes 1837) (Plate.2.e).

Distribution: The species were found inhabits holes beneath tide pools and mud habitats in the near shore areas of Nijhum Island and GPS location $22^{\circ} 02^{\prime}$ $30.6^{\prime \prime} \mathrm{N}, 90^{\circ} 58^{\prime} 24.5^{\prime \prime} \mathrm{E}$. Also it was recorded in Bhola, charfashion estuaries area and Pasur River is near the Sundarbans in Bagerhat. Rahman (2005) recorded maximum size is $13.3 \mathrm{~cm}$ in total length from estuaries area. This intertidal species found on sand and mud flats along bay shores (Martin et. al. 1999). Also found in the tidal zone of freshwater rivers, also recorded in Indo-West Pacific ranges from Pakistan to Japan and Australia and known from the freshwater tidal zone of the Mekong (Rainboth 1996). 
Description : Morphometric measurements are as follows, Number of Fin Rays/Spines: $1^{\text {st }}$ dorsal fin rays 7 and $2^{\text {nd }}$ dorsal fin rays 28; Pectoral Fin Rays 17; Pelvic Fin Rays 12; Anal Fin Rays 26; Caudal Fin Rays 9+9; Mouth position Sub-terminal; Branchiostegal Rays 3; Body measurement: Maximum Total Length $14.8 \mathrm{~cm}$ and standard length $11.4 \mathrm{~cm}$; Pre-Dorsal length $3.67 \mathrm{~cm}$; Body Depth $1.17 \mathrm{~cm}$; Head measurement: Eye diameter $4.5 \mathrm{~mm}$; Pre Orbital Length $6.2 \mathrm{~mm}$; Post Orbital Length $15.1 \mathrm{~mm}$; Inter Orbital Length $2.6 \mathrm{~mm}$; Fin base length: $1^{\text {st }}$ Dorsal Fin Base length $44 \mathrm{~mm}$; Pectoral Fin Base Length $7.3 \mathrm{~mm}$ and Pectoral Fin length $13.3 \mathrm{~mm}$; Pelvic Fin base length $4.7 \mathrm{~mm}$ and Pelvic Fin length $12.9 \mathrm{~mm}$; Anal fin Base length $56.5 \mathrm{~mm}$ (Plate.2.e).

Body color is greenish, becoming lighter below side and elongate, subcylindrical anteriorly. Amphibious air-breather fish, first dorsal fin is tall and mast-like, Some black spots widely separated upon the head, body and dorsal fins, Second dorsal widely separated from the first, Scales are rudimentary, mouth nearly horizontal and teeth in one row on each jaw, Caudal barred with black.

Meristic measurements are as follows: $\mathrm{SL} / \mathrm{TL}=0.770 ; \mathrm{HL} / \mathrm{SL}=0.249$; Pelvic Fin length $(\mathrm{PEL}) / \mathrm{SL}=0.113 ; \mathrm{PEL} / \mathrm{HL}=0.454 ;$ Pectoral Fin length $(\mathrm{PEC}) / \mathrm{SL}=$ $0.116 ; \mathrm{PEC} / \mathrm{HL}=0.397 ; \mathrm{PEC} / \mathrm{PEL}=1.031$; Body Depth $(\mathrm{BD}) / \mathrm{SL}=0.102$; PreDorsal length $(\mathrm{PDL}) / \mathrm{SL}=0.321$; Inter Orbital Length $(\mathrm{IOL}) / \mathrm{SL}=$ 0.022(Plate.2.e).

\section{Apocryptes bato (Hamilton 1822)}

Local Name: Chewa Bele, Chiring; English Name: Goby

Taxonomic position: Phylum: Chordata-chordates; Class: Actinopterygii-ray finned fishes, spiny rayed fishes; Order: Perciformes- perch like fishes; Family: Gobiidae - true gobies, gobies, Genus: Apocryptes-Valenciennes in Cuvier and Valenciennes 1837, Species: Apocryptes bato (Hamilton, 1822) (Plate.2.f).

Distribution: The species were found inhabits muddy bottoms of estuaries area of Hatiya $22^{\circ} 17^{\prime} 28.4^{\prime \prime} \mathrm{N} \& 91^{\circ} 08^{\prime} 27.0^{\prime \prime} \mathrm{E}$; Dhal char, Char fashon, Bhola; Pasur River is near the Sundarbans in Bagerhat. Rahman (2005) recorded maximum size is $15.4 \mathrm{~cm}$ in total length from Dakatia and Meghna River near Chadpur in Bangladesh.

Description : Morphometric measurements are as follows, Number of Fin Rays/Spines: $1^{\text {st }}$ dorsal fin rays 5 and $2^{\text {nd }}$ dorsal fin rays 21; Pectoral Fin Rays 23; Pelvic Fin Rays 12; Anal Fin Rays 22; Caudal Fin Rays 10+10; Mouth position Sub-terminal; Branchioostegal Rays 4/3; Scales Along Lateral Line 6080; Body measurement: Maximum Total Length $13 \mathrm{~cm}$ and standard length 8.6 $\mathrm{cm}$; Pre-Dorsal length $2.5 \mathrm{~cm}$; Body Depth $1.12 \mathrm{~cm}$; Peduncle Length $0.52 \mathrm{~cm}$; Peduncle Depth $0.7 \mathrm{~cm}$. Head measurement: Eye diameter $3.6 \mathrm{~mm}$; Pre Orbital Length $4.5 \mathrm{~mm}$; Post Orbital Length $9.2 \mathrm{~mm}$; Inter Orbital Length $1.8 \mathrm{~mm}$; Fin base length: $1^{\text {st }}$ Dorsal Fin Base length $13.7 \mathrm{~mm}$; $2^{\text {nd }}$ Dorsal Fin Base length $41.3 \mathrm{~mm}$; Pectoral Fin Base Length $5.9 \mathrm{~mm}$ and Pectoral Fin length $10.8 \mathrm{~mm}$; 
Pelvic Fin base length $3.8 \mathrm{~mm}$ and Pelvic Fin length $10.8 \mathrm{~mm}$; Anal fin Base length $35.7 \mathrm{~mm}$ (Plate.2.f).

Body color is greenish above and below side whitish, Pelvic and anal colorless. Dorsal fin higher than the anal fin, Caudal lanceolate, body elongate, posteriorly compressed Scales cycloid and small, Mouth nearly horizontal, teeth notched.

Meristic measurements are as follows: $\mathrm{SL} / \mathrm{TL}=0.661 ; \mathrm{HL} / \mathrm{SL}=0.222$; Pelvic Fin length $(\mathrm{PEL}) / \mathrm{SL}=0.125 ; \mathrm{PEL} / \mathrm{HL}=0.565 ;$ Pectoral Fin length $(\mathrm{PEC}) / \mathrm{SL}=$ $0.125 ; \mathrm{PEC} / \mathrm{HL}=0.565 ; \mathrm{PEC} / \mathrm{PEL}=1.00$; Body Depth $(\mathrm{BD}) / \mathrm{SL}=0.130$; PreDorsal length $(\mathrm{PDL}) / \mathrm{SL}=0.290$; Inter Orbital Length (IOL)/SL $=0.020$ (Plate.2.f).

\section{Boleophthalmus boddarti (Pallas 1770)}

Local Name: Dahuk; $\quad$ English Name: Blue-spotted Mudskipper

Taxonomic position: Phylum: Chordata-chordates; Class: Actinopterygii-ray finned fishes, spiny rayed fishes; Order: Perciformes- perch like fishes; Family: Gobiidae - true gobies, gobies, Genus: Boleophthalmus Valenciennes in Cuvier and Valenciennes (1837), Species: Boleophthalmus boddarti (Pallas 1770) (Plate.2.g).

Distribution: The species were found inhabits muddy bottoms at estuaries area of Nijhum Dwip (Island) and GPS location $22^{\circ} 02^{\prime} 30.6^{\prime \prime} \mathrm{N}, 90^{\circ} 58^{\prime} 24.5^{\prime \prime} \mathrm{E}$. Hatiya $22^{\circ} 17^{\prime} 28.4^{\prime \prime} \mathrm{N} \& 91^{\circ} 08^{\prime} 27.0^{\prime \prime} \mathrm{E}$; Dhal char, Charfashon, Bhola; Chuna river $22^{\circ} 16^{\prime} 20.1^{\prime \prime N} \& 89^{\circ} 11^{\prime} 51.6^{\prime \prime} \mathrm{E}$; Pasur River is near the Sundarbans in Bagerhat. Rahman 2005 recorded maximum size is $10.1 \mathrm{~cm}$ in total length from estuary areas. It was also recorded in Indo-West Pacific: India to New Guinea and north to China (Rainboth 1996). Also recorded from Sulaibikhat Bay, Persian Gulf (Wright 1988).

Description: Morphometric measurements are as follows, Number of Fin Rays/Spines: $1^{\text {st }}$ dorsal fin rays 5 and $2^{\text {nd }}$ dorsal fin rays 20; Pectoral Fin Rays 18; Pelvic Fin Rays 12; Anal Fin Rays 24; Caudal Fin Rays 9+9; Mouth position Sub-terminal; Branchiostegal Rays 3; Body measurement: Maximum Total Length $7.17 \mathrm{~cm}$ and standard length $5.78 \mathrm{~cm}$; Pre-Dorsal length $2.3 \mathrm{~cm}$; Body Depth $7.6 \mathrm{~cm}$; Peduncle Length $0.3 \mathrm{~cm}$; Peduncle Depth $0.41 \mathrm{~cm}$. Head measurement: Eye diameter $3.5 \mathrm{~mm}$; Pre Orbital Length $4.2 \mathrm{~mm}$; Post Orbital Length $9.5 \mathrm{~mm}$; Inter Orbital Length $1.7 \mathrm{~mm}$; Fin base length: 1st Dorsal Fin Base length $3.1 \mathrm{~mm}$ and $1^{\text {st }}$ Dorsal Fin length $141 \mathrm{~mm} ; 2^{\text {nd }}$ Dorsal Fin Base length $24.5 \mathrm{~mm}$; Pectoral Fin Base Length $5.3 \mathrm{~mm}$ and Pectoral Fin length 11 $\mathrm{mm}$; Pelvic Fin base length $3.7 \mathrm{~mm}$ and Pelvic Fin length $9.3 \mathrm{~mm}$; Anal fin Base length $22.9 \mathrm{~mm}$ (Plate.2.g).

Body color laterally is brown to greenish with 7 or 8 black bands; ventrally whitish to grey, head and body covered with bluish spot; $1^{\text {st }}$ dorsal fin much higher than the body and greenish numerous small pale blue speckles, $2^{\text {nd }}$ dorsal membrane greenish with bluish spots between rays; eyes above the level of their orbits; Scales cycloid; Caudal fin symmetrical and brawn to dark grey. 
Meristic measurements are as follows: $\mathrm{SL} / \mathrm{TL}=0.806$; HL/SL $=0.326$; Pelvic Fin length $(\mathrm{PEL}) / \mathrm{SL}=0.160 ; \mathrm{PEL} / \mathrm{HL}=0.492 ;$ Pectoral Fin length $(\mathrm{PEC}) / \mathrm{SL}=$ $0.190 ; \mathrm{PEC} / \mathrm{HL}=0.582 ; \mathrm{PEC} / \mathrm{PEL}=1.182$; Body Depth $(\mathrm{BD}) / \mathrm{SL}=1.314$; PreDorsal length $(\mathrm{PDL}) / \mathrm{SL}=0.397$; Inter Orbital Length (IOL)/SL $=0.029$ (Plate.2.g).

Acknowledgements: Authors are grateful to acknowledge the 'Faunal Survey and Enrichment of Facilities for Biodiversity Research Sub-project' (CP 2216), HEQEP, UGC for the financial support.

\section{LITERATURE CITED}

AKIHITO, IKEDA Y. and SUGIYAMA, K. 2002. Gobioidei. In "Fishes of Japan with Pictorial Keys to the Species, English Edition" Ed by T Nakabo, Tokai University Press, Tokyo, pp 1139-1310, 1596-1619.

ALLEN, M. J. 1991. Beam-trawl survey of bay and nearshore fishes of the soft-bottom habitat of southern California in 1989. Calif. Coop. Oceanic Fish. Invest. Rept., 32:112-127.

BIRDSONG, R.S. and ROBINS, C.R. 1995. New genus and species of seven-spined goby (Gobiidae: Gobiosomini) from the offing of the Amazon River, Brazil. Copeia 1995, 676-683.

HERRE A.W. 1927. Gobies of the Philippines and the China Sea. Monogr Bur Sci Manila 23: $1-352$.

IUCN Bangladesh. 2000. Red Book of Threatened Fishes of Bangladesh. IUCN-The World Conservation Union. Xii+116pp.

JORDAN DS and HUBBS CL 1925. Record of fishes obtained by David Starr Jordan in Japan, 1922. Mem Carnegie Mus 10: 93-346, pls. 5-12

JONES G and VAN PARIJS SM. 1993. Bimodal echolocation in pipistrelle bats: are cryptic species present? Proc R Soc London Ser B Biol Sci 251: 119-125.

KURITA, TAKAKI and YOSHINO, TETSUO 2012. Cryptic Diversity of the Eel Goby, Genus Taenioides (Gobiidae: Amblyopinae), in Japan. Zoological Science 29: 538-545.

MCDOWALL RM. and FRANKENBERG RS 1981. The galaxiid fishes of Australia (Pisces: Galaxiidae). Rec Aust Mus 33: 443-605.

MARTIN, K.L.M. and C.R. BRIDGES, 1999. Respiration in water and air. p. 54-78. In M.H. Horn, K.L.M. Martin and M.A. Chotkowski (eds.) Intertidal fishes. Life in two worlds. Academic Press. 399 p.

MURDY, E.O. and SHIBUKAWA, K. 2001. A revision of the gobiid fish genus Odontamblyopus (Gobiidae: Amblyopinae). Ichthyol Res 48: 31-43.

MURDY, E.O. and RANDALL, J.E. 2002. Taenioides kentalleni, a new species of eel goby from Saudi Arabia (Gobiidae: Amblyopinae). Zootaxa 93: 1-6

NELSON, J.S. 2006. Fishes of the World. John Wiley and Sons, New York. 4th edition.419-424 pp.

RAINBOTH, W.J. 1996. Fishes of the Cambodian Mekong. FAO species identification field guide for fishery purposes. FAO, Rome, $265 \mathrm{p}$. 
RAHMAN, A.K. A. 2005. Freshwater Fishes of Bangladesh, 2nd ed., Zool. Soc. Bangladesh, Dhaka, Bangladesh, xvii+394pp.

RUBER, L.,VAN TASSELL, J.L. and ZARDOYA, R. 2003. Rapid speciation and ecological divergence in the American seven-spined gobies (Gobiidae, Gobiosomatini) inferred from a molecular phylogeny. Evolution 57: 1584-1598.

SHEN, G.Y. 2008. Gobiidae: Amblychaeturichthys, Chaeturichthys, Eutaeniichthys, Lophiogobius, Odontamblyopus, Parachaeturichthys, Taenioides. In "Fauna Sinica, Ostichthyes, Perciformes (V), Gobioidei” Ed by HL Wu, JS Zhong, Science Press, Beijing, 740-743pp.

STERBA, G. 1962. Freshwater Fishes of the World. Vista Books, London. 847 pp.

KURITA,T. and YOSHINO,T 2012. Cryptic Diversity of the Eel Goby, Genus Taenioides (Gobiidae: Amblyopinae), in Japan. Zoological Science, 29(8):538-545. http://dx.doi.org/10.2108/zsj.29.538

TALWAR, P.K. and JHINGRAN, A.G. 1991. Inland Fishes of India and Adjacent Countries. Vol. 1 \& 2. Oxford \& IBH Publishing Co. Pvt. Ltd., New Delhi, India. 1158 pp.

TOMIYAMA, I. 1936. Gobiidae of Japan. Japan J Zool 10: 37-112.

WRIGHT, J.M., 1988. Seasonal and spacial differences in the fish assemblage of the nonestuarine Sulaibikhat Bay, Kuwait. Mar. Biol. 100:13-20.

(Manuscript received on 24 June, 2015; revised on 3 November, 2015) 